\title{
Sinusoidal Efflux of Glutathione in the Perfused Rat Liver Evidence for a Carrier-mediated Process
}

Murad Ookhtens, Karen Hobdy, Morrow C. Corvasce, Tak Yee Aw, and Neil Kaplowitz Liver Research Laboratory, Medical and Research Services, Veterans Administration Wadsworth Medical Center and the University of California, Los Angeles, California 90073

\begin{abstract}
Turnover of hepatic glutathione in vivo in the rat is almost entirely accounted for by cellular efflux, of which $80-90 \%$ is sinusoidal. Thus, sinusoidal efflux plays a major quantitative role in homeostasis of hepatic glutathione. Some preliminary observations from our laboratory (1983. J. Pharmacol. Exp. Ther. 224:141-147.) and circumstantial evidence in the literature seemed to imply that the raising of the hepatic glutathione concentration above normal was not accompanied by a rise in the rate of sinusoidal efflux. Based on these observations, we hypothesized that the sinuisoidal efflux was probably a saturable process and that at normal levels of hepatic glutathione the effux behaved as a zero-order process (near-saturation). We tested our hypothesis by the use of isolated rat livers perfused in situ, single pass, with hemoglobin-free, oxygenated buffer medium at pH $7 . \dot{4}$ and $37^{\circ} \mathrm{C}$. Preliminary experiments established a range of perfusion rates $(3-4 \mathrm{ml} / \mathrm{min}$ per $\mathrm{g}$ ) for adequacy of oxygenation, lack of cell injury, and minimization of variability contributed by perfusion rates. Hepatic glutathione was lowered to below normal by a 48 -h fast, diethylmaleate (0.1-1.0 ml/kg i.p:), and buthionine sulfoximine $(8 \mathrm{mmol} / \mathrm{kg}$ i.p.), and raised to above normal by 3-methylcholanthrene (20 $\mathrm{mg} / \mathrm{kg} \times 3 \mathrm{~d}$ i.p.) and cobalt chloride $\left(0.05-0.27 \mathrm{~g} / \mathrm{kg}^{-1} \mathrm{sub}-\right.$ cutaneously). Steady state sinusoidal efflux from each liver was measured over a 1-h perfusion, during which the coefficient of variation of glutathione in perfusates stayed within $10 \%$. Hepatic glutathione efflux as a function of hepatic concentration was characterized by saturable kinetics with sigmoidal (nonhyperbolic) features. The data were fitted best with the Hill model and the following parameter values were estimated: $V_{\max }$ $=20 \mathrm{nmol} / \mathrm{min}$ per $\mathrm{g}, K_{\mathrm{m}}=3.2 \mu \mathrm{mol} / \mathrm{g}$, and $n=3 \mathrm{binding} /$ transport sites. The efflux could be inhibited reversibly by sulfobromophtalein-glutathione conjugate but was not affected by the addition of glutathione to the perfusion medium. The results support our hypothesis that sinusoidal efflux of glutathione is near saturation ( $\simeq \mathbf{8 0 \%}$ of $V_{\max }$ ) at normal (fed and fasted) liver glutathione concentrations. The phenomenon of saturability coupled with the ability to inhibit the efflux leads us to propose that sinusoidal efflux from hepatocytes appears
\end{abstract}

This work was presented in preliminary form at the 34th Annual Meeting of the American Association for the Study of Liver Diseases, Chicago, 4 and 5 November 1983 and has been published in abstract (1983. Hepatology [Baltimore]. 3:325).

Address reprint requests to Dr. Ookhtens, Liver Research Laboratory, Building 115, Room 316, VA Wadsworth Medical Center, Los Angeles, CA 90073.

Received for publication 14 February 1984 and in revised form 18 September 1984.

The Journal of Clinical Investigation, Inc.

Volume 75, January 1985, 258-265 to be a carrier-mediated process. Some recent studies by others, using sinusoidal membrane-enriched vesicles, also support these conclusions.

\section{Introduction}

Glutathione, a tripeptide ( $\gamma$-glutamylcysteinylglycine), plays a key role in numerous physiological regulatory processes and in detoxification reactions in the hepatocyte $(1,2)$, where it exists almost entirely in the reduced form (GSH). ${ }^{1}$ Efflux of hepatic GSH constitutes a major component of the homeostatic regulation of GSH in the liver, since it accounts for at least 85 to $90 \%$ of the total cellular turnover of this tripeptide under normal conditions (3-5). The efflux of GSH takes place into both sinusoidal blood and bile (2). However, the sinusoidal efflux is by far the major component and constitutes from $\mathbf{8 0}$ to $90 \%$ of the total efflux (4). Therefore, sinusoidal efflux plays a quantitatively significant role in the total hepatic GSH turnover and homeostasis.

Although characteristics of the biliary efflux of hepatic GSH have been studied in vivo (4) and in canalicular membrane preparations (6), little is known about the nature and quantitative regulation of sinusoidal efflux. Some circumstantial evidence from our and other investigators' studies with perfused livers and isolated hepatocytes $(4,7,8)$ implied that the sinusoidal efflux of GSH might take place with uniform rates over a range of intracellular GSH concentrations near that of a fed rat. In addition, a GSH transport system has been proposed from studies with hepatic sinusoidal plasma membrane-enriched vesicles (9). However, it is not known whether this transport system is involved in the sinusoidal efflux of hepatic GSH under physiological conditions.

To gain an understanding of the nature of the sinusoidal efflux of GSH from the intact liver, we studied the kinetics of this process using isolated rat livers perfused in situ. The aim of our study was to test the hypothesis that the mechanism of hepatic GSH efflux may be functioning near capacity or at saturation (zero-order process) in the livers of fed rats. We perturbed the liver GSH concentrations to near steady states below and above that in the fed state in order to define the kinetics of the sinusoidal efflux of GSH as a function of hepatic GSH concentration.

\section{Methods}

Chemicals. GSH, GSH reductase, glyoxalase I (lactoyl-glutathione lyase), and NADPH were obtained from Sigma Chemical Co. (St.

1. Abbreviations used in this paper: BSO, buthionine sulfoximine; BSP, sulfobromophthalein; DEM, diethylmaleate; GSH, glutathione; 3-MC, 3-methylcholanthrene; $V_{\mathrm{C}}$ and $V_{\mathrm{EC}}$, liver intracellular and extracellular fluid volumes, respectively. 
Louis, MO). All other materials used were of $A R$-grade and were readily available from commercial sources.

Tracers and scintillation spectrometry. [Carboxyl- ${ }^{14} \mathrm{C}$ ]inulin, 2.6 $\mu \mathrm{Ci} / \mathrm{mg}$, 98\% pure, and ${ }^{3} \mathrm{H}_{2} \mathrm{O}$ (lots 1496-149 and 909-189, respectively) were obtained from New England Nuclear, Boston, MA). The radioactivity in perfusates and liver homogenates or protein-precipitated samples were measured by liquid scintillation spectrometry on a Beckman counter (model LS-3150T; Beckman Instruments, Inc., Fullerton, CA). Appropriate quench monitoring and corrections were made.

Animals. Male Sprague-Dawley rats (Hilltop Lab Animals, Inc., Scottdale, PA), 200-300 g, were used. The animals had free access to water and Purina rodent chow (Ralston Purina Co., St. Louis, MO), unless they were to be studied in the fasted state. In the latter case, food was removed $48 \mathrm{~h}$ before study, causing a 16\% loss in body weight. The livers of all rats except those in the fasted group averaged $\sim 9 \mathrm{~g}$. In the fasted group they averaged $\sim 6 \mathrm{~g}$. All rats were anesthetized with an intraperitoneal injection of pentobarbital $(50 \mathrm{mg} / \mathrm{kg})$ before the perfusions (see Isolated liver perfusions, below).

\section{Treatments}

To alter the hepatic GSH concentration to below and above that of fed rats, the following treatments were used. The three used to lower hepatic GSH were as follows.

48- $h$ fast. The effect of such a prolonged fast is a well-established reduction in the hepatic GSH concentration by $\sim 30 \%$ from fed level $(10,11)$. In our rats, the hepatic GSH dropped from $5.7 \pm 0.2 \mu \mathrm{mol} / \mathrm{g}$ (mean \pm SE, $n=25)$ to $4.0 \pm 0.2(n=10)$, consistent with earlier reports.

Diethylmaleate (DEM). Intraperitoneal injection of this compound causes the depletion of hepatic GSH by formation of a GSH conjugate (12), which is excreted into bile. The excretion is presumably completed $1 \mathrm{~h}$ after the injection, as evidenced by the reported transient rise in the hepatic bile flow and fall to normal levels in $1 \mathrm{~h}$ (13). Our preliminary tests established that depressed levels of GSH reached $1 \mathrm{~h}$ after DEM injection were maintained for the additional hour studied. We used doses of DEM between 0.1 and $1.0 \mathrm{ml} / \mathrm{kg}$ to obtain a range of liver GSH concentrations below that of fed rats for our kinetic studies.

Buthionine sulfoximine (BSO). This compound is a potent inhibitor of GSH synthesis. It inhibits the conversion of cysteine to $\gamma$-glutamyl cysteine (14). We synthesized BSO according to the method described by Griffith (15). We standardized our BSO dose to $8 \mathrm{mmol} / \mathrm{kg}$ i.p. ( $(4$ $\mathrm{ml}$ of a $0.5 \mathrm{M}$ solution of $\mathrm{BSO}$ in $0.9 \% \mathrm{NaCl}$ ) in order to lower liver GSH concentrations. The results of an initial time study, when the above dose was used (data not shown), established that the hepatic GSH concentration was depleted, with a $t_{1 / 2}$ of $\sim 3 \mathrm{~h}$ up to $\sim 6 \mathrm{~h}$; a nadir was then reached at about $8 \mathrm{~h}$, after which the GSH returned to normal by the next day ( $24 \mathrm{~h})$. Thus, GSH concentrations as low as $\simeq 1 \mu \mathrm{mol} / \mathrm{g}$ could be attained by this treatment. For our kinetic studies, the livers were isolated and perfused (for up to $1 \mathrm{~h}$ ) at different intervals after the BSO injections. The postperfusion liver GSH concentrations were very similar to those obtained in the in vivo time study, indicating that near-steady state conditions were maintained during perfusions.

Three types of treatments were tried, two of which were used to raise the hepatic GSH content for our kinetic studies.

Phenobarbital. This compound has been used to induce a rise in hepatic GSH $(4,16)$. Doses of $80 \mathrm{mg} / \mathrm{kg}$ per $\mathrm{d} \times 3 \mathrm{~d}$ i.p. (given in two injections per day, morning and evening) were used, which raised the hepatic GSH concentration to $6.7 \pm 0.1(n=4) \mu \mathrm{mol} / \mathrm{g}$. Although the mean liver GSH level of the phenobarbital-treated rats was significantly above the mean of the fed group $(P<0.05)$, considerable overlap existed between the two groups. Therefore, instead of phenobarbital, other treatments were chosen to raise the hepatic GSH concentrations to a level substantially above that of fed rats.

3-Methylcholanthrene (3-MC). We have used this compound earlier to raise the hepatic GSH concentration (4). In our present study, we injected 3-MC dissolved in corn oil $(20 \mathrm{mg} / \mathrm{ml})$ i.p. $(20 \mathrm{mg} / \mathrm{kg} \times 3 \mathrm{~d}$, morning and evening). A pair of control rats injected with comparable amounts of corn oil alone had liver GSH concentration and efflux similar to those of untreated, fed rats (data not shown). 3-MC treatment caused a significant rise $(P<0.001)$ in the mean hepatic GSH concentration as compared with that of controls, i.e., fed rats (7.6 $\pm 0.2 \mathrm{SE}, n$ $=8$ vs. $5.7 \pm 0.2, n=25 \mu \mathrm{mol} / \mathrm{g})$.

Cobalt chloride $\left(\mathrm{CoCl}_{2}\right)$. This compound induces a dramatic rise in the hepatic GSH, presumably by inducing $\gamma$-glutamylcysteine synthetase (17). We injected single or multiple doses of $0.05 \mathrm{~g} / \mathrm{kg} \mathrm{CoCl}$ (in saline) subcutaneously into the lower dorsal region of the rats. Doses were given twice a day, morning and evening; the last injection was always given on the evening before the day of perfusion. A range of liver GSH concentrations from $\simeq 9$ to $14 \mu \mathrm{mol} / \mathrm{g}$ could be attained by cumulative multiple doses of $\mathrm{CoCl}_{2}$ ranging between 0.05 and $0.27 \mathrm{~g} / \mathrm{kg}$.

\section{Isolated liver perfusions}

Livers from untreated (fed) and pretreated rats, as described above, were perfused in situ, single-pass, with Krebs-Ringer bicarbonate buffer gassed to equilibrium with $95 \% \mathrm{O}_{2} / 5 \% \mathrm{CO}_{2}$ at $\mathrm{pH} 7.4$ and $37^{\circ} \mathrm{C}$. The method was essentially that described by Sies (18). We continuously monitored the $\mathrm{O}_{2}$ tension in the inflow and outflow lines to determine the rate of $\mathrm{O}_{2}$ uptake by the livers. The hydrostatic perfusion pressure was also continuously monitored and remained well below 10 $\mathrm{CmH}_{2} \mathrm{O}$.

We did a series of preliminary experiments to evaluate the characteristics of our perfused liver model and to compare it with published data for validation of our system. We perfused a group of livers that had normal, subnormal, and supernormal GSH concentrations. In each perfusion, the rate of flow was varied stepwise in the range of 1$4 \mathrm{ml} / \mathrm{min}$ per $\mathrm{g}$, and GSH efflux was determined over a 15 -min interval for each flow rate. The results showed that as the perfusion rates reached $\geq 3 \mathrm{ml} / \mathrm{min}$ per $\mathrm{g}$ the rate of efflux rose to a plateau and thereafter remained independent of further increases in the perfusion rate. Similarly, the average $\mathrm{O}_{2}$ uptake rose to plateau values of $>1.5$ $\mu \mathrm{mol} / \mathrm{min}$ per $\mathrm{g}$. Thus, we set a perfusion rate of $\geq 3 \mathrm{ml} / \mathrm{min}$ per $\mathrm{g}$ and an $\mathrm{O}_{2}$ uptake $>1.5 \mu \mathrm{mol} / \mathrm{min}$ per $\mathrm{g}$ as criteria for acceptability of any perfusion. Besides these parameters, the absence of hepatic cell injury was ascertained by the absence of glutathione-S-transferase activity (a cytosolic enzyme) in the perfusates.

Perfusions to define the kinetics of hepatic GSH efflux were conducted for $\simeq 1 \mathrm{~h}$ beyond an initial 10-min equilibration after the onset of the perfusions. The loss of hepatic GSH concentration after a 1-h perfusion was minor as compared with that in similarly treated but unperfused livers. Thus, adequate semi-steady state conditions existed during the kinetic experiments. The existence of near-steady state conditions was also confirmed by the steady state rates of efflux observed. Eight equally spaced perfusate samples, taken in each hourlong perfusion, commonly had a coefficient of variation $<10 \%$. The mean of the eight determinations was used to calculate the rate of steady state GSH efflux in nanomoles per minute per gram.

When perfusions with sulfobromophthalein (BSP)-GSH conjugate (synthesized by the method described in reference 19), GSH, or tracers were desired, these substances were prepared as concentrated solutions (in perfusion buffer) and infused into the perfusion line far enough from the liver to ensure thorough mixing before entry into the liver. The flow rates of the infusions did not exceed $3 \%$ of the total perfusion flow. During control periods (before or after infusion of the desired substances) the side infusion was maintained with buffer alone and at rates similar to those of the infused substances.

In our kinetic studies, the perfusion rates were $3.9 \pm 0.05 \mathrm{ml} / \mathrm{min}$ per $\mathrm{g}$ (mean $\pm \mathrm{SE}, n=85$ ), perfusion pressures were $5.2 \pm 0.3 \mathrm{~cm} \mathrm{H}_{2} \mathrm{O}$ $(n=71)$, and the $\mathrm{O}_{2}$ uptakes were $2.1 \pm 0.04 \mu \mathrm{mol} / \mathrm{min}$ per $\mathrm{g}(n=85)$.

Measurement of liver extracellular and intracellular fluid volumes ( $V_{E C}$ and $V_{C}$, respectively)

To test for the possibility of an increased $V_{E C} / V_{C}$ in the livers of rats fasted for $48 \mathrm{~h}$ vs. those of fed rats (see Discussion), a number of livers 
from each group were perfused as follows. After an initial control period of perfusion, [carboxyl- ${ }^{14} \mathrm{C}$ ] inulin and ${ }^{3} \mathrm{H}_{2} \mathrm{O}$ were infused into the inflow line at a constant rate through the side $(Y)$ connection described above. The rate of infusion of tracers was set to 0.002 and $0.02 \mu \mathrm{Ci} / \mathrm{min}$ per $\mathrm{ml}$ of perfusate for $\left[{ }^{14} \mathrm{C}\right]$ inulin and ${ }^{3} \mathrm{H}_{2} \mathrm{O}$, respectively. Samples from inflow and outflow lines were taken directly for liquid scintillation spectrometry. Whole liver radioactivities were determined at the end of each perfusion, after homogenization and precipitation of the proteins. The steady state specific activities of $\left[{ }^{14} \mathrm{C}\right]$ inulin and ${ }^{3} \mathrm{H}_{2} \mathrm{O}$ in the perfusates (counts per minute per milliliter) and livers (counts per minute per gram) were used, along with the dry vs. wet weights of liver samples, to calculate the $V_{E C}$ as a percentage of the total liver water space.

\section{Assays}

Hepatic GSH efflux was measured by determination of the total GSH equivalents (reduced GSH + oxidized glutathione) in the perfusate samples by the GSH recycling assay of Tietze (20). The coefficient of variation of this assay for multiple determinations on samples with GSH concentration in the range of our perfusates $(\simeq 0.5-8 \mu \mathrm{M})$ averaged $\sim 5 \%$. Concentration differences as low as $0.5 \mu \mathrm{M}$ (or even lower) could be readily resolved at the low end of the range of values studied. Our tests on perfusate samples with the glyoxalase I method (21) indicated that essentially all of the glutathione in the perfusate samples was in the reduced form, GSH. Liver GSH was determined by the method of Owens and Belcher (22) after the proteins were precipitated from crude liver homogenates $(33 \%$, wt/vol, with $0.01 \mathrm{M}$ sodium phosphate- $0.25 \mathrm{M}$ sucrose buffer, $\mathrm{pH} 7.4$ ) with $10 \%$ trichloroacetic acid (1:1, vol/vol). Perfusate samples containing BSP-GSH conjugate were assayed for their BSP content by measurement of absorbance at $580 \mathrm{~nm}$ (19). Corrections were made for the inhibitory effect of BSP-GSH on the recycling assay.

\section{Kinetic analyses}

To select an appropriate kinetic model for estimating the $V_{\max }$ and apparent $K_{\mathrm{m}}$ of hepatic GSH efflux, we tested several standard kinetic models by attempting to fit them to our data. The fitting (nonlinear least squares) was done with the SAAM program (23) on the IBM 3033 (IBM Instruments, Inc., IBM Corp., Danbury, CT) at the Office of Academic Computing of the University of California, Los Angeles. The criteria used for goodness of fit to select the model that fitted the data best were: $(a)$ absence of systematic deviations of the fitted curves from the data, and $(b)$ minimum (relative) sum of squared errors.
In our attempts, the kinetic model that fitted the data best (with or without correction of data for the mitochondrial GSH pool; see Results and Discussion) was the Hill equation, defined as: $v=V_{\max } \cdot[\mathrm{GSH}]^{n} /\left(K+[\mathrm{GSH}]^{n}\right)$, where $K_{\mathrm{m}}=\sqrt[n]{K}$ and $n$ is the number of cooperative binding/transport sites.

Our attempts to fit models defined by one or two superimposed hyperbolas with and without additive linear terms were unsuccessful. In all cases, there were systematic deviations of the fits from the data and larger sum of squared errors as compared with that obtained with the Hill model (see Discussion).

\section{Results}

Perfused liver studies to define the kinetics of GSH efflux. Groups of livers from fed and fasted rats, as well as those from rats given various pretreatments, were perfused as described in Methods. The mean GSH efflux computed for each liver plotted against the corresponding liver GSH concentration is shown in Fig. 1. The data were characterized by a rise in the GSH efflux concurrent with a rise in the liver GSH concentration from near-fully depleted to normal (fasted and fed) values. Thereafter, the rate of efflux seems to have reached a plateau (saturation) independent of further increases in the hepatic GSH concentration. The data also seem to be characterized by sigmoidal features.

Kinetic analyses and parameter estimation. As stated in Methods, we tested a group of standard kinetic models by attempting to fit them to our data. The best fit (according to the criteria presented in Methods), represented by the curve in Fig. 1, was obtained by the use of the Hill equation and the following estimated values of kinetic parameters: $V_{\max }=20 \mathrm{nmol} / \mathrm{min}$ per $\mathrm{g}( \pm 4 \%$, coefficient of variation), $K_{\mathrm{m}}=3.2 \mu \mathrm{mol} / \mathrm{g}( \pm 5 \%)$, and $n=2.9( \pm 12 \%)$.

If we assume that our treatments to deplete the hepatic GSH pool left the mitochondrial pool essentially intact (see Discussion), our data can be modified to represent the GSH efflux vs. cytosolic GSH (modified data not shown). After this correction, the Hill model still provided the best fit, with the following parameter estimates: $V_{\max }=20 \mathrm{nmol} / \mathrm{min}$ per $\mathrm{g}$ $( \pm 5 \%), K_{\mathrm{m}}=2.5 \mu \mathrm{mol} / \mathrm{g}( \pm 6 \%)$, and $n=2.2( \pm 16 \%)$.

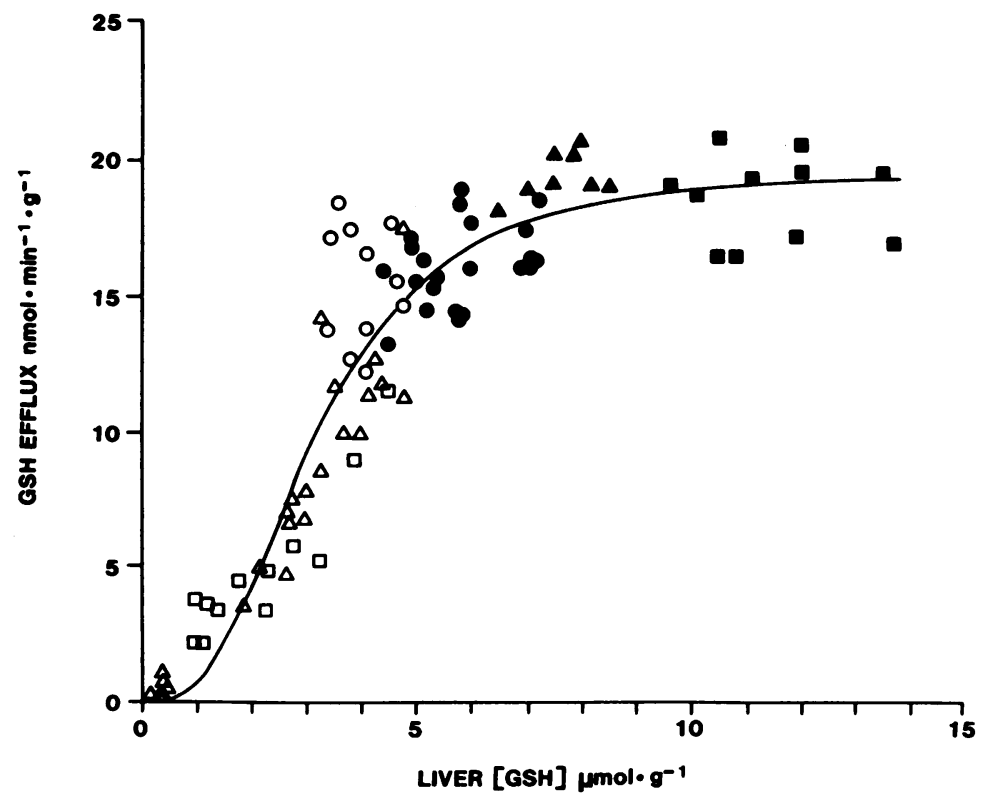

Figure 1. Data defining the kinetics of hepatic GSH efflux. Each data point represents the mean efflux of GSH from a single liver perfused over a 1-h interval. $\bullet$, fed; $\circ$, fasted for $48 \mathrm{~h} ; \Delta$, diethylmaleate; $\square$, buthionine sulfoximine; $\Delta$, 3methylcholanthrene; and $\mathrm{m}, \mathrm{CoCl}_{2}$. The curve represents the best (least-squares) fit obtained by use of the Hill model (see Results) and the SAAM program (23). All data points were weighed equally. The kinetic parameters defined by the fit were $V_{\max }=20 \mathrm{nmol} / \mathrm{min}$ per $\mathrm{g}, K_{\mathrm{m}}=3.2 \mu \mathrm{mol} / \mathrm{g}$, and $n$ $\simeq 3$. 
Comparison of fed and fasted groups. A noteworthy feature of our kinetic data is the relationship between the fed and fasted groups. When the data in Fig. 1 representing the fed and fasted livers were grouped, the picture presented in Fig. 2 emerged. The well-documented phenomenon of the fall, by $\sim 30 \%$, of the hepatic GSH concentration from fed to fasted livers was evident. However, this fall was not accompanied by a fall in the GSH efflux. Thus, the fractional rate of efflux rose significantly (by $40 \%$ ) in the fasted state as compared with the fed. This phenomenon, the apparent maintenance of the GSH efflux (mass $\cdot$ time $^{-1}$ ) in the face of a falling liver GSH content, can at least partially account for the reported (11) doubling of the fractional rate of turnover during fasting.

Because we had expressed the GSH efflux as normalized to gram wet weight of liver tissue, it occurred to us that the differences between fasted and fed groups discussed above could be an artifactual one based on the following argument. If the shifts in the $V_{\mathrm{C}}$ vs. $V_{\mathrm{EC}}$ of the fasted livers were toward a relatively larger $V_{\mathrm{EC}} / V_{\mathrm{C}}$ as compared with the fed livers, then normalizing per gram wet tissue weight would result in a relatively underestimated liver GSH content in the fasted livers. In that case, a normalization such as one expressing all concentrations per gram of cells or milliliter of $V_{C}$ would be more appropriate and would perhaps eliminate the differences described in Fig. 2.

To check for the possibility that $V_{\mathrm{EC}} / V_{\mathrm{C}}$ was increased by fasting, a group of livers from fed and fasted rats were perfused with the added infusion of $\left[{ }^{14} \mathrm{C}\right]$ inulin and ${ }^{3} \mathrm{H}_{2} \mathrm{O}$ as described in Methods. The radioactivity of both tracers in the outflow samples (counts per minute per milliliter) reached a plateau (steady state) value, equal to that of the inflow samples, within 2 min after the onset of the constant infusion of the mixture of the two tracers (data not shown). The steady state was maintained, with no apparent slow trends, for the duration of the 20-min study.

Table I presents the summary of the results (after plateau was reached). As can be seen, there were no significant differences between the $V_{\mathrm{EC}}$ of fed and fasted groups.

Inhibition of GSH efflux by BSP-GSH conjugate. A group of rat livers were perfused with BSP-GSH conjugate (19). In these perfusions, after an initial control period of $30 \mathrm{~min}$
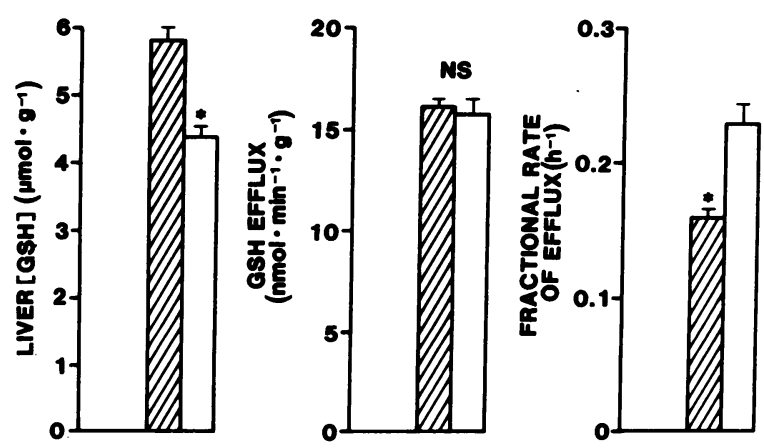

Figure 2. Comparison of the fed (ㅁ) and fasted (ㅁ) groups presented in Fig. 1. The left panel shows the significant fall (by $30 \%$ ) of hepatic GSH concentration induced by a $48-\mathrm{h}$ fast. The middle panel shows the equality of the mean efflux under both conditions. The right panel shows the significant rise (by $40 \%$ ) of the fractional rate of efflux in the fasted as compared with the fed group. ${ }^{*} P<0.001$. during which the livers were perfused with buffer, a constant step infusion of BSP-GSH was started and maintained for $\mathbf{3 0}$ min; the BSP-GSH infusion was then stopped and followed with a 30-min perfusion with buffer alone. The efflux of GSH in response to the BSP-GSH conjugate decreased and remained at a plateau from 15 to $30 \mathrm{~min}$ after the onset of BSP-GSH infusion. This inhibitory effect of BSP-GSH conjugate was reversible, and 15-20 min after the BSP-GSH step was turned off (recovery period) GSH efflux was usually back to the preBSP-GSH or control levels. The plateau values of GSH efflux between 15 and $30 \mathrm{~min}$ after the onset of BSP-GSH steps were used to compute the percentage inhibition (as compared with the control period) of GSH efflux. Table II presents the summary of the data from four perfusions conducted in this way. As can be seen, an almost $30 \%$ inhibition in the GSH efflux was obtained with a concentration of $\simeq 10 \mu \mathrm{M}$ BSPGSH conjugate.

Effect of perfusion with GSH on efflux. To test for the possibility of transstimulation or inhibition of GSH efflux by GSH, we did a group of perfusions in which we infused GSH into the inflow line (see Methods). A concentration range of ingoing GSH between 10 and $40 \mu \mathrm{M}$ did not have any effect on the GSH efflux (measured as net difference between inflow and outflow GSH).

\section{Discussion}

Although characteristics of the biliary efflux of glutathione have been examined in some detail $(4,6)$, only the recent study by Inoue et al. (9) on sinusoidal membrane-enriched vesicles addresses the problem of sinusoidal GSH transport. Efflux of biliary glutathione in the oxidized form occurs against a 50-fold concentration gradient (active transport) (24). This transport accounts for only $\sim 30 \%$ of the total biliary efflux (25); we found that $70 \%$ of the biliary efflux was in the form of GSH (25). Biliary efflux of GSH was down a two- to sixfold concentration gradient (5-7 $\mu \mathrm{mol} / \mathrm{g}^{-1}$ or $\mathrm{mM}$ liver to $1-2 \mathrm{mM}$ bile), and the kinetics defined in our laboratory showed a proportional (linear) relationship between the biliary GSH efflux and liver GSH concentration, with no tendency for saturation at liver GSH concentrations as high as $8 \mu \mathrm{mol} / \mathrm{g}(4$, and unpublished observations). This work was recently confirmed by others $(26)$. Inoue et al. $(6,27)$ have described a carrier for GSH in canalicular membrane preparations with $K_{\mathrm{m}}=0.33 \mathrm{mM}$. However, the absence of saturation in biliary GSH efflux in vivo suggests that this carrier may not be responsible for the bulk of biliary GSH excretion.

Sinusoidal efflux of glutathione, almost all in the form of GSH, occurs down a much larger ( $>100$-fold) concentration gradient (5-7 $\mu \mathrm{mol} / \mathrm{g}$ or $\mathrm{mM}$ liver to $30-40 \mu \mathrm{M}$ hepatic vein plasma). Some preliminary evidence from perfused rat livers $(4,7)$ and isolated hepatocytes $(8)$ had indicated that when hepatic GSH was raised above normal by phenobarbital treatment or varied in the physiologic range, no apparent concurrent change took place in the efflux. These observations prompted us to hypothesize that the process of efflux is perhaps at or near saturation in a liver with normal GSH concentrations. Therefore, we designed experiments with isolated, in situperfused livers to test this hypothesis.

We used at least two different agents to lower or raise the hepatic GSH concentration because we were concerned about the possible effects of any one of these treatments on the 
Table I. Comparison of $V_{E C}$ and $V_{C}$ in Livers from Fed Rats and Rats Fasted for $48 h$

\begin{tabular}{|c|c|c|c|c|c|c|c|c|c|c|c|}
\hline \multirow[b]{2}{*}{$\begin{array}{l}\text { Nutritional } \\
\text { state }\end{array}$} & \multicolumn{2}{|c|}{ Body weight } & \multirow[b]{2}{*}{$\begin{array}{l}\text { Liver } \\
\text { weight }\end{array}$} & \multirow{2}{*}{$\begin{array}{l}\text { Liver } \\
\text { weight/ } \\
\text { body } \\
\text { weight }\end{array}$} & \multirow[b]{2}{*}{$\begin{array}{l}\text { Perfusion } \\
\text { rate }\end{array}$} & \multirow[b]{2}{*}{$\begin{array}{l}\mathrm{O}_{2} \\
\text { uptake }\end{array}$} & \multirow[b]{2}{*}{$\begin{array}{l}\text { Liver } \\
\text { [GSH] }\end{array}$} & \multirow[b]{2}{*}{$\begin{array}{l}\text { Liver } \\
\text { dry } \\
\text { weight* }\end{array}$} & \multirow[b]{2}{*}{$\begin{array}{l}\text { Liver } \\
\text { water } \\
\text { spaceł }\end{array}$} & \multirow[b]{2}{*}{$V_{\mathrm{EC}} \S$} & \multirow[b]{2}{*}{$V_{\mathbf{c}^{\prime \prime}}$} \\
\hline & $\begin{array}{l}\text { Before } \\
\text { fasting }\end{array}$ & $\begin{array}{l}\text { After } \\
\text { fasting }\end{array}$ & & & & & & & & & \\
\hline & $g$ & $g$ & $g$ & $\%$ & $\begin{array}{l}\mathrm{ml} / \mathrm{min} \\
\text { per } \mathrm{g}\end{array}$ & $\begin{array}{l}\text { umol/min } \\
\text { per } g\end{array}$ & $\mu \mathrm{mol} / \mathrm{g}$ & $\begin{array}{l}\text { \% of } \\
\text { wet wt }\end{array}$ & $\begin{array}{l}\text { \% of } \\
\text { wet wt }\end{array}$ & $\begin{array}{l}\text { \% of } \\
\text { water } \\
\text { space }\end{array}$ & $\begin{array}{l}\text { \% of } \\
\text { water } \\
\text { space }\end{array}$ \\
\hline \multicolumn{12}{|l|}{ Fed } \\
\hline$(n=4)$ & \multicolumn{2}{|c|}{$289 \pm 20$} & $10.4 \pm 0.3$ & $3.5 \pm 0.1$ & $4.1 \pm 0.1$ & $2.0 \pm 0.1$ & $6.5 \pm 0.3$ & $26 \pm 0.3$ & $79 \pm 4.4$ & $18 \pm 2.3$ & $82 \pm 1.0$ \\
\hline $\begin{array}{c}\text { Fasted for } \\
48 \mathrm{~h}\end{array}$ & & & & & & & & & & & \\
\hline$(n=3)$ & $307 \pm 10$ & $273 \pm 11$ & $6.8 \pm 0.4$ & $2.5 \pm 0.06$ & $4.2 \pm 0.3$ & $2.1 \pm 0.1$ & $4.1 \pm 0.2$ & $26 \pm 0.8$ & $72 \pm 5.0$ & $16 \pm 0.9$ & $84 \pm 0.9$ \\
\hline
\end{tabular}

All values are mean \pm SE. * Measured gravimetrically by drying aliquots of liver homogenates. $¥$ Measured from the difference between ${ }^{3} \mathrm{H}_{2} \mathrm{O}$ in perfusates and liver. As can be seen, the sum of the liver water space, traced by ${ }^{3} \mathrm{H}_{2} \mathrm{O}$, and dry weight (percentage of wet weight), measured gravimetrically, was $100 \%$. This implies both that the mixing of ${ }^{3} \mathrm{H}_{2} \mathrm{O}$ in the liver total water space had been accomplished and that the recovery of the tracer measurements was $100 \%$. Therefore, no correction to the $\left[{ }^{14} \mathrm{C}\right]$ inulin data was necessary. $§$ Measured from the difference between $\left[{ }^{14} \mathrm{C}\right.$ ]inulin in perfusates and livers. each liver.

mechanism of GSH efflux. DEM depletes the hepatic GSH pool by forming a conjugate that has to be disposed of (into bile); therefore, it could possibly inhibit the transport of GSH by competition for a common carrier and thereby cause the measurement of an apparent lower efflux in livers depleted of GSH by DEM. We delayed the onset of the perfusion of the livers of DEM-treated rats to at least $1 \mathrm{~h}$ post-DEM injection. By that time the conjugated DEM-GSH had presumably been disposed of, as evidenced by the transient rise and fall of the rate of bile secretion (13). However, to rule out the possibility that an inhibitory effect of DEM-GSH conjugate might have been responsible for the apparent depressed efflux of GSH in DEM-depleted livers, we used BSO also. This substance causes the depletion of the hepatic GSH pool by inhibition of synthesis at the step of the conversion of cysteine to $\gamma$ glutamylcysteine (14). Thus, presumably, no substances that could compete with GSH efflux would be present in livers of rats treated with BSO.

The rates of efflux measured in livers with GSH concentrations raised above those of livers fed by 3-MC treatment (Fig. 1) already show the tendency toward saturation and seem to rule out a linear kinetic relationship. However, a much higher range of liver GSH concentration needed to be explored to establish unequivocally that the process of sinusoidal efflux was saturable. These higher GSH concentrations were obtained by $\mathrm{CoCl}_{2}$ treatment. We were also concerned that $\mathrm{CoCl}_{2}$ treatment might be able to lower the GSH efflux by an inhibitory mechanism, resulting in the appearance of a pseu- dosaturation. That such a pseudosaturation had not occurred was verified in a separate experiment. When the hepatic GSH concentration of livers from seven $\mathrm{CoCl}_{2}$-treated rats was lowered to near-fed levels by DEM injections $(1 \mathrm{~h}$ before perfusion), GSH effluxes no different from those in four control (normal, fed) livers used for this study were obtained (data not shown). Thus, regardless of the way the treatments were used (even two different ones in the same animal), the kinetics of efflux seemed to be consistent with the results shown in Fig. 1. We also examined the possibility that $\mathrm{CoCl}_{2}$ might exert a direct effect on the transport mechanisms by membrane depolarization or some other nonspecific toxicity. No change in the rate of $\mathrm{Na}^{+}$-dependent uptake of amino acids in hepatocytes isolated from $\mathrm{CoCl}_{2}$-treated rats as compared with controls was observed (unpublished observations).

Besides the foregoing, there was also a complete absence of any apparent adverse effects on the livers by any of the agents and treatments used. This was verified by the consistency and uniformity of parameters such as $\mathrm{O}_{2}$ uptake, perfusion pressure, and the absence of enzyme release in the livers from treated and untreated groups. The viability of hepatocytes treated with the different agents was checked in the isolated cell systems and found to be similar to that of the untreated ones by trypan blue exclusion and the absence of differential cell injury determined by enzyme release (unpublished observations). Finally, the livers from all pretreated groups, similar to those from untreated ones (fed and fasted), maintained steady effluxes for the $\sim 1$-h duration of each perfusion (coef-

Table II. Inhibition of Net Hepatic GSH Efflux by BSP-GSH Conjugate in Perfused Rat Livers*

\begin{tabular}{|c|c|c|c|c|c|c|}
\hline \multirow[b]{2}{*}{ Liver [GSH] } & \multicolumn{2}{|c|}{ [BSP-GSH] } & \multirow[b]{2}{*}{ BSP-GSH uptake } & \multicolumn{2}{|l|}{ Net GSH efflux } & \multirow[b]{2}{*}{ Inhibition } \\
\hline & In & Out & & Controlł & BSP-GSH & \\
\hline$\mu \mathrm{mol} / \mathrm{g}$ & $\mu M$ & $\mu M$ & $n$ mol/min per $g$ & nmol/min per $g$ & nmol/min per $g$ & \% of control \\
\hline $5.6 \pm 0.3 \S$ & $9.7 \pm 1.2$ & $7.4 \pm 1.0$ & $9.5 \pm 1.8$ & $16.0 \pm 0.9$ & $11.4 \pm 0.6$ & $29 \pm 3$ \\
\hline
\end{tabular}

* Livers used in this experiment had $\mathrm{O}_{2}$ uptake $(1.9 \pm 0.1 \mu \mathrm{mol} / \mathrm{min}$ per $\mathrm{g})$ and hydrostatic perfusion pressure $\left(4.0 \pm 0.3 \mathrm{~cm} \mathrm{H}_{2} \mathrm{O}\right)$ similar to those of the livers used in our kinetic studies. There was also no evidence for liver cell injury as monitored by the absence of cytosolic enzyme, glutathione $S$-transferase, activity in the perfusates (see text for details of the design). $¥$ Control: pre-BSP-GSH efflux. $§ \mathrm{Mean} \pm \mathrm{SE}, n=4$. 
ficient of variation for eight samples taken over the observation interval usually $<10 \%$ ).

Based on the above discussions, the possibility that our treatments to raise or lower hepatic GSH above or below levels in fed livers affected the mechanism of GSH transport remains low; however, it cannot be totally ruled out. More prolonged treatments, such as maintenance of rats on a selenium-deficient diet, have been observed to cause a substantially higher efflux of GSH than normal from hepatocytes and perfused livers (8). However, the possibility that during such prolonged treatments induction of the carrier takes place and/ or its properties are altered cannot be ruled out.

Our kinetic data (Fig. 1) support the hypothesis that the sinusoidal efflux of GSH is near saturation (zero-order process) in the range of normal concentrations of hepatic GSH. In fact, in the range of mean hepatic GSH concentrations in livers of rats fasted for $48 \mathrm{~h}$ to concentrations in livers of fed rats (4.0 \pm 0.2 to $5.7 \pm 0.2 \mu \mathrm{mol} / \mathrm{g}$ liver), the sinusoidal efflux averaged $16 \mathrm{nmol} / \mathrm{min}$ per $\mathrm{g}$ liver, which is $\sim 80 \%$ of the $V_{\max }\left(V_{\max }\right.$ $=20 \mathrm{nmol} / \mathrm{min}$ per $\mathrm{g}$ liver) determined by our kinetic analysis (see below).

Our attempts to find a kinetic model that was consistent with the features of our data and fitted them best led us to select the Hill model. Note that our choice of this model was totally ad hoc and based solely on its utility in terms of bestfitting the sigmoidal shape of our data (which are to a large extent preserved even when corrected by subtraction for an estimated mitochondrial pool). Our primary purpose for the use of the Hill model was the estimation of kinetic parameters. Two of these parameters, $V_{\max }=20 \mathrm{nmol} / \mathrm{min}$ per $\mathrm{g}$ and apparent $K_{\mathrm{m}}=3.2 \mu \mathrm{mol} / \mathrm{g}$, can be fairly readily determined (by the use of an eye-fitted curve) from the kinetic data without the use of any particular model. The third parameter, $n$, which is adjusted to $\simeq 3$ by the iterations during fitting, poses a dilemma. There is no a priori reason to expect a multisite carrier for GSH with cooperativity between the transport sites. However, this remains as one possibility predicted by our modeling, provided that systematic errors of unknown origin have not generated the sigmoidal features of our kinetic data. We must also stress that although the model we have used is the simplest formulation that can fit sigmoidal kinetics, it is not unique in this respect; at this juncture, however, a more exhaustive modeling exercise is unwarranted because of the absence of additional definitive data to discriminate among the choices of several different models. Inoue et al. (9) in some recent studies of GSH transport with hepatic sinusoidal membrane-enriched vesicles have suggested two saturable carrier systems for transport of GSH: low and high affinity with $K_{\mathrm{m}}=3.3$ and $0.34 \mathrm{mM}$, respectively. It is interesting that our estimated $K_{\mathrm{m}}, 3.2 \mu \mathrm{mol} / \mathrm{g}(\simeq \mathrm{mM})$ is identical to the $K_{\mathrm{m}}$ of their low-affinity carrier. Taken together, these two results provide strong evidence for the existence of this carrier and its involvement in the sinusoidal efflux of hepatic GSH in the intact organ. Whether the high-affinity carrier Inoue et al. observed is also involved in the efflux of hepatic GSH from the intact organ could not be determined from our data. It was not possible to obtain satisfactory fits to our data with the sum of two hyperbolic functions, with or without subtraction of the mitochondrial pool of GSH (the second hyperbolic term consistently was adjusted to zero by the iterations). The only way we could fit two superimposed functions to our data were by using the sum of a Hill function and a hyperbola. However, in these trials, although minor improvements in the fits occurred, the reliability of the estimates of kinetic parameters were poor (high coefficients of variation computed by SAAM), such that the apparent $K_{m}$ of the hyperbolic function could not be resolved from zero.

Our kinetic analysis and interpretation of the data are based on some inherent assumptions which are worth delineation. To begin with, we initially treated the hepatic GSH pool as a kinetically (i.e., temporally) homogeneous pool. Lauterberg and Mitchell (11), presenting tracer-kinetic data obtained in vivo, have argued for the behavior of hepatic GSH as a single, kinetically homogeneous pool. The only inhomogeneity reported so far has been that caused by the distinction of the mitochondrial from the cytosolic pools of GSH. Meredith and Reed (28) have shown that this spatially (anatomically) distinct pool, accounting for $\simeq 10 \%$ of the total GSH in the hepatocyte, is temporally (kinetically) distinct; i.e., whereas $t_{1 / 2}$ of the cytosolic GSH turnover is $\simeq 2 \mathrm{~h}$, that of the mitochondrial pool is $\simeq 30 \mathrm{~h}$. Thus, the mitochondrial pool appears to be metabolically distinct from the cytosolic one, and its relative contribution to the cellular GSH efflux is probably negligible. We tested the effect of this inhomogeneity on our kinetic analysis and interpretations by use of the following assumptions. There is evidence that DEM may deplete the cytosolic pool of GSH selectively and not affect the mitochondrial GSH (28). In the case of BSO, the mechanism of depletion is by inhibition of synthesis; therefore, the large difference in the $t_{1 / 2}$ of the cytosolic $(\simeq 2 \mathrm{~h})$ vs. mitochondrial $(\simeq 30 \mathrm{~h})$ turnover would cause a selectively higher depletion of the cytosolic pool, leaving the mitochondrial pool essentially intact during the short periods of BSO treatment that we used (1 to $8 \mathrm{~h}$ ). In addition, fasting might deplete the mitochondrial pool. Thus, when either DEM or BSO is used to deplete the hepatic GSH, the mitochondrial pool that is to be subtracted from the total to obtain the cytosolic pool is probably equal to that in a fed liver. Using this correction did not significantly change the results of our kinetic analysis. Similarly, if we assume that there was as much as two-thirds depletion of the mitochondrial pool by fasting, the correction did not change the sigmoidal shape of the data. Thus, the mitochondrial corrections will not change the estimates of $V_{\max }$ but will shift the $K_{\mathrm{m}}$ to a lower value.

We also verified experimentally that the reason for the fasting group's lower total hepatic GSH concentration (30\% lower than for the fed group) was not an artifactual one caused by our expressing the liver GSH concentrations per gram of wet tissue. Thus, we observed no preferential fluid volume shifts from the cellular to the extracellular in the fasted livers to warrant any correction. However, the possibility remains that normalization per weight of wet tissue in the fasted liver introduces an error if the loss of liver weight is not correlated with the loss of number of carrier units. Such a lack of correlation is, for example, possible if the bulk of liver weight loss in our fasted livers is due to glycogen depletion.

There is the possibility also of other spatial inhomogeneities of the GSH pool in the liver. Lobular gradients of GSH have been observed with histochemical methods (29). The existence of such gradients would render our analysis as a grossly lumped, first-approximation approach to defining the kinetic parameters of sinusoidal GSH efflux from the hepatocyte. The degree and extent of these inhomogeneities will be instrumental in revealing the accuracy of our estimates of the kinetic 
parameters obtained by a lumped approach and model. More detailed and refined studies will be required to clarify these issues.

An additional observation that supports the likelihood of the involvement of a carrier-mediated mechanism in hepatic GSH efflux was the inhibitory effect of the BSP-GSH conjugate. There was a latency of $\sim 15 \mathrm{~min}$ both for inhibited efflux to reach steady state and for the reversal of the inhibitory effect. This implies that the BSP-GSH had to be taken up to exert its inhibitory effect from the cytoplasmic side. Nevertheless, whether or not the observed inhibitory effect of BSP-GSH is a competitive one for a common carrier mechanism remains to be explored. It would require the perfusion of a number of livers having low to high GSH concentrations with BSP-GSH conjugate to see whether at high GSH concentrations the inhibitory effect is overcome. The specificity of the transport mechanism for a certain class of molecules with some common physicochemical properties would be additional support for the existence of a carrier mechanism. At this point, the qualitative observation of inhibition of GSH efflux by a structurally related molecule (BSP-GSH) supports the possibility that a carrier-mediated process has been inhibited. In a concurrent study in our laboratory with freshly isolated hepatocyte incubations and very similar kinetics (i.e., sigmoidal and saturable), we have observed a dramatic and reversible inhibitory effect of methionine on the GSH efflux (30). Whether this inhibition is exerted by competition for a common carrier also remains to be studied. However, these findings indicate that sinusoidal efflux of GSH is a regulated process. We also perfused a number of livers from fed rats with elevated levels of GSH in the perfusion medium. Our aim was to explore whether GSH concentrations in the range of 10-40 $\mu \mathrm{m}$ (equivalent to that in the hepatic portal vein of fed rats). would have any transstimulatory or inhibitory effect on the carrier mechanism. No effect on the GSH efflux (net across the liver) could be established.

Removal of GSH circulating in blood has been thought to occur only by its breakdown to cysteine by $\boldsymbol{\gamma}$-glutamyltranspeptidase, a brush-border enzyme in the renal and intestinal tissues, and uptake in that form (31). There is some direct evidence that the uptake of GSH as an intact molecule can occur in the kidney (32). Such an uptake has not been demonstrated in the liver (33). However, the overwhelming electrochemical and concentration gradients in favor of the efflux of hepatic GSH might make it difficult to measure uptake of GSH even when labeled extracellular GSH is used.

In conclusion, we have shown that the process of the sinusoidal efflux of hepatic GSH is saturable and that it can be inhibited. At the normal range of hepatic GSH concentrations (fasted to fed) the rate of this efflux is near $(\sim 80 \%)$ the $V_{\max }$ of the transport process. The efflux begins to fall only with more severe depletion than that obtained by fasting (such as that caused by DEM and BSO) perhaps to conserve hepatic GSH. Since sinusoidal efflux of hepatic GSH comprises the largest part, i.e., 70-85\%, of the degradative component of total intrahepatic turnover (total efflux accounts for $85-90 \%$ of total hepatic GSH turnover, the sinusoidal component for 80-90\% of total efflux) it has a major role in the dynamic regulation and moment-to-moment maintenance of the homeostasis of intrahepatic GSH. The $t_{1 / 2}$ of vascular GSH turnover is very short (on the order of a few minutes) (33) and GSH is removed rapidly, mostly by the renal and intestinal tissues (14). It has been proposed that the liver, by supplying circulating GSH, acts as a reservoir of cysteine for the rest of the tissues (10) or regulates thiol-disulfide status of membrane and plasma proteins. Our significant finding is that in the normal state of hepatic function the efflux of hepatic GSH takes place at rates near capacity or saturation $\left(V_{\max }\right)$ of this process. In fact, even a 48-h fast, which causes a $30 \%$ depletion of the hepatic GSH concentration, does not cause a perceptible change in the rate of efflux. We can only speculate that purpose of the maintenance of efflux at near-capacity rates is to ensure an adequate vascular supply of GSH, probably for a vital function (such as those mentioned or some others) as far as the rest of the organism is concerned. Whether the turnover of vascular GSH serves such a proposed vital function or is a futile cycle remains to be seen.

\section{Acknowledgments}

We gratefully acknowledge Dr. Ajit Arora for his valuable assistance in synthesizing the BSO, and Mr. John Kuhlenkamp for his technical assistance at various phases of this study. The secretarial assistance of Ms. Anita Boesman is greatly appreciated.

This work was supported by National Institutes of Health grant AM 30312 and Veterans Administration research funds. Dr. Corvasce was supported by National Institutes of Health Training Grant AM 07180 .

\section{References}

1. Kosower, N. D., and E. M. Kosower. 1978. The glutathione status of cells. Int. Rev. Cytol. 54:104-160.

2. Kaplowitz, N. 1981. The importance and regulation of heptic glutathione. Yale J. Biol. Med. 54:497-502.

3. Bartoli, G. A., and H. Sies. 1978. Reduced and oxidized glutathione efflux from liver. FEBS (Fed. Eur. Biochem. Soc.) Lett. 86:89-91.

4. Kaplowitz, N., D. E. Eberle, J. Petrini, J. Touloukian, M. C. Corvasce, and J. Kuhlenkamp. 1983. Factors influencing the efflux of hepatic glutathione into bile in rats. J. Pharmacol. Exp. Ther. 224:141147.

5. Lauterburg, B. H., J. D. Adams, and J. R. Mitchell. 1983. Efflux of glutathione (GSH) from the liver accounts for total hepatic production in vivo. Fed. Proc. 42:890.

6. Inoue, M., R. Kinne, T. Tran, and I. M. Arias. 1983. The mechanism of biliary secretion of reduced glutathione, analysis of transport process in isolated rat-liver canalicular membrane vesicles. Eur. J. Biochem. 134:467-471.

7. Sies, H., G. M. Bartoli, R. F. Burk, and C. Waydhas. 1978. Glutathione efflux from perfused rat liver after phenobarbital treatment during drug oxidations, and in selenium deficiency. Eur. J. Biochem. 89:113-118.

8. Hill, K. E., and R. F. Burk. 1983. Effect of methionine and cysteine on glutathione synthesis by selenium-deficient isolated rat hepatocytes. In Functions of Glutathione: Biochemical, Physiological, Toxicological and Clinical Aspects. A. S. Larsson, S. Orrenius, A. Holmgren, and B. Mannervik, editors. Raven Press, New York. 117 124.

9. Inoue, M., R. Kinne, T. Tran, and I. M. Arias. 1984. Glutathione transport across hepatocyte plasma membranes: analysis using isolated rat liver sinusoidal-membrane vesicles. Eur. J. Biochem. 138:491-495.

10. Tateishi, N., T. Higashi, A. Naruse, K. Nakashima, H. Shiozaki, and Y. Sakamoto. 1977. Rat liver glutathione: possible role as a reservoir of cysteine. J. Nutr. 107:51-60.

11. Lauterburg, B. H., and J. R. Mitchell. 1981. Regulation of hepatic glutathione turnover in rats in vivo and evidence for kinetic homogeneity of the hepatic glutathione pool. J. Clin. Invest. 67:14151424. 
12. Boyland, E., and L. F. Chasseaud. 1970. The effect of some carboxyl compounds on rat liver glutathione levels. Biochem. Pharmacol. 19:1526-1528.

13. Barnhart, J., and B. Combes. 1979. Chloresis associated with metabolism of biliary excretion of diethylmaleate in the rat and dog. J. Pharmacol. Exp. Ther. 206:614-623.

14. Griffith, O. W., and A. Meister. 1979. Glutathione: interorgan translocation, turnover, and metabolism. Proc. Natl. Acad. Sci. USA. 76:5606-5610.

15. Griffith, O. W. 1981. Depletion of glutathione by inhibition of biosynthesis. Methods Enzymol. 77:59-63.

16. Kaplowitz, N., J. Kuhlenkamp, L. Goldstein, and J. Reeve. 1980. Effect of salicylates and phenobarbital on hepatic glutathione in the rat. J. Pharmacol. Exp. Ther. 212:240-245.

17. Sasame, H. A., and M. R. Boyd. 1978. Paradoxical effects of cobaltous chloride and salts of other divalent metals on tissue levels of reduced glutathione and microsomal mixed-function oxidase components. J. Pharmacol. Exp. Ther. 205:718-724.

18. Sies, H. 1978. The use of perfusion of liver and other organs for the study of microsomal electron transport and cytochrome P-450 system. Methods Enzymol. 51:48-59.

19. Whelan, G., J. Hoch, and B. Combes. 1970. A direct assessment of the importance of conjugation for biliary transport of sulfobromophtalein sodium. J. Lab. Clin. Med. 75:542-557.

20. Tietze, F. 1969. Enzymic method for quantitative determination of nanogram amounts of total and oxidized glutathione: applications to mammalian blood and other tissues. Anal. Biochem. 27:502-522.

21. Racker, E. 1951. The mechanism of action of glyoxalase. $J$. Biol. Chem. 190:685-696.

22. Owens, C. W. I., and R. V. Belcher. 1965. A colorimetric micro-method for the determination of glutathione. Biochem. J. 94:705711.

23. Berman, M., and M. F. Weiss. 1977. SAAM Manual. Department of Health, Education and Welfare, National Institutes of Health,
Government Printing Office, Washington, DC. Publication No. 76730.

24. Akerboom, T. P. M., M. Bilzer, and H. Sies. 1982. The relationship of biliary glutathione disulfide efflux and intracellular glutathione disulfide content in perfused rat liver. J. Biol. Chem. 257:4248-4252.

25. Eberle, D., R. Clarke, and N. Kaplowitz. 1981. Rapid oxidation in vitro of endogenous and exogenous glutathione in bile of rats. $J$. Biol. Chem. 256:2115-2117.

26. Lauterburg, B. H., C. V. Smith, H. Hughes, and J. R. Mitchell. 1984. Biliary excretion of glutathione and glutathione disulfide in the rat. Regulation and response to oxidative stress. J. Clin. Invest. 73: 124-133.

27. Inoue, M., T. Tran, R. Kinne, and I. M. Arias. 1982. Translocation of hepatic glutathione into bile and plasma: mechanism of glutathione transport by isolated rat liver sinusoidal and canalicular plasma membrane vesicles. Hepatology. 2:720. (Abstr.)

28. Meredith, M. J., and D. J. Reed. 1982. Status of the mitochondrial pool of glutathione in the isolated hepatocyte. J. Biol. Chem. 257:3747-3753.

29. Asghar, K., B. G. Reddy, and G. Krishna. 1975. Histochemical localization of glutathione in tissues. J. Histochem. Cytochem. 23:774779.

30. Aw, T. Y., M. Ookhtens, and N. Kaplowitz. 1984. Inhibition of glutathione efflux from isolated rat hepatocytes by methionine. $J$. Biol. Chem. 259:9355-9358.

31. Meister, A. 1983. Selective modification of glutathione metabolism. Science (Wash. DC). 220:472-477.

32. Lash, L. H., and D. P. Jones. 1983. Transport of glutathione by renal basal-later membrane vesicles. Biochem. Biophys. Res. Commun. 112:55-60.

33. Hahn, R., A. Wendel, and F. L. Flohe. 1978. The fate of extracellular glutathione in the rat. Biochim. Biophys. Acta. 539:324337. 